\title{
Cumulative burden of disease: a relevant measure of the late side-effects of cancer treatment
}

\author{
MC Aznar1,3, S Darby1, GP Collins4, D Cutter1,2 \\ 1.Clinical Trial Service Unit, Nuffield Department of Population Health, University of Oxford, \\ Oxford, UK, \\ 2. Department of Oncology, Oxford Cancer and Haematology Centre, University of Oxford, \\ Oxford, UK, \\ 3. Department of Oncology, Rigshospitalet, Copenhagen University Hospital, Copenhagen, \\ Denmark, \\ 4. Department of Clinical Haematology, Oxford Cancer and Haematology Centre, University \\ of Oxford, Oxford, UK
}

Children, teenagers, and young adults diagnosed with Hodgkin's lymphoma a decade ago had an excellent prognosis, with 10-year overall survival above 90\%. ${ }^{1}$ For those diagnosed today, outcomes should be even better through efforts to prevent side-effects by reducing treatment as far as possible without compromising cure. In The Lancet Oncology, a report by Nickhill Bhakta and colleagues ${ }^{2}$ from the St Jude Lifetime Cohort Study (SJLIFE) shows that, in 10-year survivors of Hodgkin's lymphoma diagnosed between 1961 and 2004 who reached at least 18 years of age, treatment-related cardiovascular morbidity ranged from subclinical to life-threatening, with new manifestations continuing to appear during several decades of follow-up. In 670 participants from SJLIFE, at 50 years of age, the cumulative incidence of survivors experiencing at least one grade 3-5 cardiovascular condition was $45.5 \%$ (95\% Cl 36.6-54.3), compared with 15.7\% (7.0-24.4) in age and sex frequency-matched community controls. These risks are known to depend on the treatments given (chemotherapy or radiotherapy) and are also strongly affected by age, sex, lifestyle factors (eg, smoking and exercise), and other cardiovascular risk factors (eg, hypertension and diabetes). The strengths of SJLIFE are the large size of the cohort for a relatively rare disease such as Hodgkin's lymphoma and the long-term prospective follow-up in the St Jude Long-term Follow-up Study, with direct clinical assessment possible for many patients.

Bhakta and colleagues ${ }^{2}$ propose a novel method for quantifying the total cardiovascular burden of young survivors of Hodgkin's lymphoma treated in the past by considering all the cardiovascular conditions observed so far in the patient's lifetime. Their approach might be relevant not just for this example but also for many other cancer diagnoses and late side-effects. Furthermore, assessment of the continuing cumulative burden of treatmentrelated morbidity is relevant not only from a clinical viewpoint but also from a health economics perspective. A patient experiencing an asymptomatic grade 2 condition as a first cardiovascular event, followed years later by grade 3 cardiomyopathy, will see their life expectancy and quality affected much more by the latter than the former.

In addition to providing a useful measure of the consequences so far of treatments given in the past, the information contained in cohorts with long- 
term follow-up remains the best available evidence that we have on which to base predictions of the future risks of late side-effects from presently used treatment strategies. Inevitably, the treatments received by the individuals in such cohorts are outdated. For example, in the St Jude Cohort, patients received old-fashioned radiotherapy, using high prescribed doses and large radiation fields, resulting in substantial doses to the heart. However, nowadays patients with Hodgkin's lymphoma receive mediastinal irradiation only if there is evidence of mediastinal disease and, even then, average heart doses would be about 7 Gy as opposed to doses of above 15 Gy for most of the St Jude cohort patients. 3 and 4 Similar considerations apply to cardiotoxic chemotherapy agents. Although doxorubicin remains in common use, the number of cycles and cumulative dose are now adapted to fit the patient's disease risk profile and response to treatment, which is often assessed using functional imaging. ${ }^{5}$

To assist with predicting future risk from contemporary radiotherapy regimens with the generally lower cardiac doses used today, the radiation doseresponse relationships for different heart diseases that have recently been derived might be helpful. 6,7 and 8 However, these dose-response relationships are based just on the first cardiovascular event and they vary both in slope and shape between different heart diseases (valvular heart disease, ischaemic heart disease, and heart failure). Further work would therefore be needed to derive an appropriate dose relationship for the cumulative burden of all cardiac morbidity for use in a prediction model. If these challenges can be overcome, the cumulative burden method could provide a valuable way of predicting the future risks of late effects when comparing treatment strategies to decide the best risk-benefit balance for today's patients with by cancer. Predictions based on the cumulative disease burden might also provide an appropriate method for assessing the socioeconomic cost of progressive chronic heart conditions when evaluating the potential effect of using novel, and hopefully less toxic, cancer treatments. However, some of the new treatments proposed are expensive. For example, proton radiotherapy has been suggested as an alternative to standard photon-based radiotherapy for some patients because it promises to reduce the exposure of healthy tissue and hence the risk of late side-effects, including cardiovascular disease and second malignancies. As of today, proton radiotherapy is still substantially more expensive than photon-based radiotherapy and access remains limited either by the number of proton treatment facilities in the patient's country or by reimbursement issues. ${ }^{9}$ Similarly, new systemic treatments, such as brentuximab vedotin and immune checkpoint inhibitors, which can be used to reduce the need for radiotherapy, are prohibitively expensive for many healthcare systems. Prioritising access to these treatments requires reliable clinical data on their effectiveness and relevant predictions of the future risks they might present (or avoid) to quantify the impact for each individual patient.

1. W Dorfell, U Ruhl, H Luders, et al.

Treatment of children and adolescents with Hodgkin lymphoma without radiotherapy for patients in complete remission after chemotherapy: final results of the multinational trial GPOH-HD95J Clin Oncol, 31 (2013), pp. 1562-1568

2. Bhakta N, Liu Q, Yeo F, et al. Cumulative burden of cardiovascular morbidity in paediatric, adolescent, and young adult survivors of Hodgkin's lymphoma: an analysis from the St Jude 
Lifetime Cohort Study. Lancet Oncol 2016; published online July 25. http://dx.doi.org/10.1016/ S1470-2045(16)30215-7.

3. Maraldo MV, Jørgensen M, Brodin NP, et al. The impact of involved node, involved field and mantle field radiotherapy on estimated radiation doses and risk of late effects for pediatric patients with Hodgkin lymphoma. Pediatr Blood Cancer 2014; 61: 717-22.

4. Girinsky T, van der Maazen R, Specht L, et al. Involved-node radiotherapy (INRT) in patients with early Hodgkin lymphoma: concepts and guidelines. Radiother Oncol 2006; 79: 270-77.

5. Cutter DJ, Schaapveld M, Darby SC, et al. Risk of valvular heart disease after treatment for Hodgkin lymphoma. J Natl Cancer Inst 2015; 107: djv008.

6. J Radford, T Illidge, N Counsell, et al.Results of a trial of PET-directed therapy for early-stage Hodgkin's Iymphoma N Engl J Med, 372 (2015), pp. 1598-1607

7. van Nimwegen FA, Schaapveld M, Cutter D, et al. Radiation dose-response relationship for risk of coronary heart disease in survivors of Hodgkin lymphoma. J Clin Oncol 2016; 34: 235-43.

8. Chow EJ, Chen Y, Kremer LC, et al. Individual prediction of heart failure among childhood cancer survivors. J Clin Oncol 2015; 33: 394-402.

9. AM Crellin, NG Burnet Proton beam therapy: the context, future direction and challenges become clearer Clin Oncol, 26 (2014), pp. 736-738 